\title{
Observed and simulated seasonal co-variations of outgoing longwave radiation spectrum and surface temperature
}

\author{
Yi Huang ${ }^{1}$ and V. Ramaswamy ${ }^{2}$ \\ Received 2 June 2008; revised 23 July 2008; accepted 5 August 2008; published 4 September 2008.
}

[1] We analyze the seasonal variations of Outgoing Longwave Radiation (OLR) accompanying the variations in sea surface temperature (SST) from satellite observations and model simulations, focusing on the tropical oceans where the two quantities are strikingly anti-correlated. A spectral perspective of this "super-greenhouse effect" is provided, which demonstrates the roles of water vapor line and continuum absorptions at different altitudes and the influences due to clouds. A model-satellite comparison indicates that the GFDL General Circulation Model can fairly well represent the total-sky radiative response to SST in the water vapor infrared absorption band despite the significant bias in the mean state, but this comprises compensating water vapor- and cloud-related errors. The analysis also reveals that the GCM significantly underestimates the cloud induced radiative responses in the window region which arises from the model bias in the mean cloud forcing in convectively active regions. Thus, spectral decomposition proves essential to understand and assess the OLR-SST relationship and the impacts of water vapor and cloud upon this linkage. Citation: Huang, Y., and V. Ramaswamy (2008), Observed and simulated seasonal co-variations of outgoing longwave radiation spectrum and surface temperature, Geophys. Res. Lett., 35, L17803, doi:10.1029/2008GL034859.

\section{Introduction}

[2] Following the climate perturbation analysis by Wetherald and Manabe [1988], the surface temperature change $\left(\Delta T_{s}\right)$ upon reaching a new equilibrium climate state, after linearization, is proportional to the initial topof-the-atmosphere (TOA) energy budget perturbation $(\Delta \mathrm{F})$. This can be expressed through

$$
\Delta \mathrm{T}_{\mathrm{s}}=\Delta \mathrm{F} \cdot\left(\frac{\partial \mathrm{F}}{\partial \mathrm{T}_{\mathrm{s}}}+\sum \frac{\partial F}{\partial \mathrm{X}_{\mathrm{i}}} \frac{\partial \mathrm{X}_{\mathrm{i}}}{\partial \mathrm{T}_{\mathrm{s}}}\right)^{-1} \cdots,
$$

where $\frac{\partial \mathrm{F}}{\partial \mathrm{T}_{\mathrm{s}}}$ denotes the radiative damping of the surface warming according to Planck's Law, $\sum \frac{\partial \mathrm{F}}{\partial \mathrm{X}_{\mathrm{i}}} \frac{\partial \mathrm{X}_{\mathrm{i}}}{\partial \mathrm{T}_{\mathrm{s}}}$ summarizes the responses in various climate variables to surface temperature change and the consequent impacts on the radiation fields, and $\left(\frac{\partial \mathrm{F}}{\partial \mathrm{T}_{\mathrm{s}}}+\sum \frac{\partial F}{\partial \mathrm{X}_{\mathrm{i}}} \frac{\partial \mathrm{X}_{\mathrm{i}}}{\partial \mathrm{T}_{\mathrm{s}}}\right)^{-1}$ quantifies the overall climate sensitivity.

\footnotetext{
${ }^{1}$ Atmospheric and Oceanic Sciences Program, Princeton University, Princeton, New Jersey, USA.

${ }^{2}$ NOAA Geophysical Fluid Dynamics Laboratory, Princeton, New Jersey, USA.
}

Copyright 2008 by the American Geophysical Union. 0094-8276/08/2008GL034859
[3] As reviewed by Bony et al. [2006], the large spread of model simulated climate feedbacks, expressed through $\sum \frac{\partial \mathrm{F}}{\partial \mathrm{X}_{\mathrm{i}}} \frac{\partial \mathrm{X}_{\mathrm{i}}}{\partial \mathrm{T}_{\mathrm{s}}}$ in the above formulation, causes a correspondingly large discrepancy in the overall sensitivity and thus climate projections. To constrain model sensitivity against observations becomes necessary and urgent. However, when using observed variations of the radiation energy budget for this purpose, difficulties often arise due to the small relative change of the broadband TOA radiation fluxes [Tsushima and Manabe, 2001; Clement and Soden, 2005], which is largely due to compensating effects that can arise from different spectral bands, or different dynamical regimes (e.g., ascending vs. descending) [Bony et al., 1997]. Moreover, as shown by Huang et al. [2007b], for the outgoing longwave radiation (OLR), compensating errors related to water vapor and cloud distributions can lead to seemingly good agreement in the broadband outgoing irradiance flux. Thus, we are motivated to spectrally decompose the relationship between OLR and surface temperature, in order to understand how different spectral bands contribute to the overall OLR sensitivity, and hence to verify the climate model in respects beyond the broadband flux. Several studies have applied spectrally resolved radiance measurements to diagnose model simulations [e.g., Iacono and Clough, 1996; Haskins et al., 1997; Huang et al., 2002, 2007b], this paper extends the previously works to an examination of the co-variations of OLR and SST under both clear- and total-skies.

[4] In this study, the "super-greenhouse effect" (SGE) in tropical oceans is our prime focus. The SGE consists of strong water vapor and cloud variations accompanying SST variation such that there results an anti-correlation between OLR and $\mathrm{T}_{\mathrm{s}}$ [Ramanathan and Collins, 1991]. The limited availability of the spectral observation enables us to investigate a regional OLR- $\mathrm{T}_{\mathrm{S}}$ relationship (the term in parenthesis in equation (1)) in the context of the seasonal cycle, whereas note that equation (1) is usually in reference to global means at multi-decadal and longer time scales. We present a spectral evaluation of SGE by examining satelliteobserved and model-simulated co-variations of OLR and SST over a seasonal cycle, analyze the roles of water vapor and clouds based on their distinctive spectral signatures, and conclude by discussing the implications of the OLR-T relationship analyzed in the SGE context.

\section{Data and Model}

[5] The observed OLR spectra employed are from the Atmospheric Infrared Sounder (AIRS) [Chahine et al., 2006] onboard Aqua which has been operational since August 2002. After eliminating noisy channels, 1026 out of the 1862 AIRS channels from 650 to $1650 \mathrm{~cm}^{-1}$ are used 


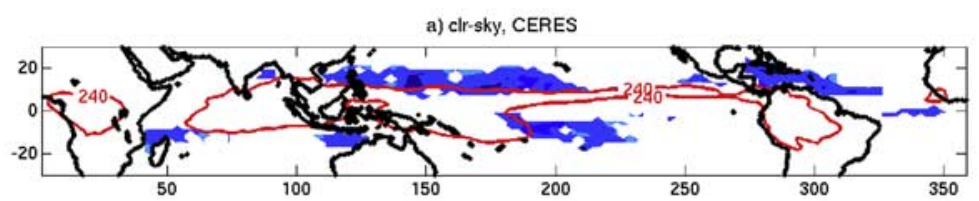

b) all-sky, CERES

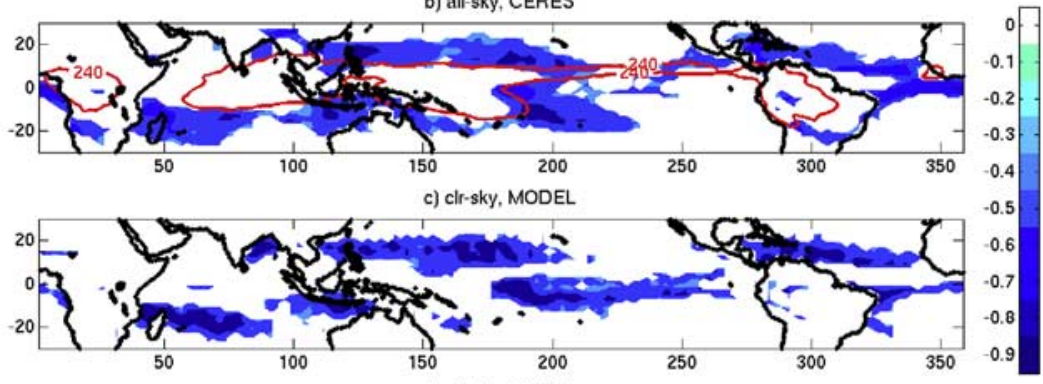

d) allsky, MODEL

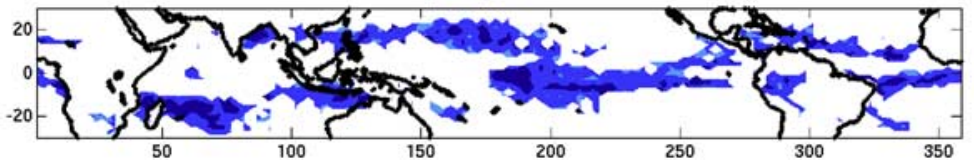

Figure 1. (a) Clear-sky and (b) total-sky SGE regions (shaded in blue, indicating significant anti-correlation between OLR and $\mathrm{T}_{\mathrm{s}}$ ) based on CERES observations. Correlation coefficient between OLR and $\mathrm{T}_{\mathrm{s}}$ is color-shaded; the regions circled by the red line have annual mean total-sky OLR less than $240 \mathrm{~W} \mathrm{~m}^{-2}$. (c) and (d) The counterparts of Figures 1a and 1b, respectively, from model simulation results.

here. Only "nadir views" ( scan angles within $\pm 5^{\circ}$ ) from the "Level-1 B" and "Level-2" radiance products are used for total-sky and clear-sky analyses respectively. For broadband irradiance fluxes, the single scanner footprint product [Loeb et al., 2007] of the Clouds and the Earth's Radiant Energy System (CERES) instrument onboard the same spacecraft is used. The sea surface temperature (SST) is taken from the NCEP reanalysis [Kalnay et al., 1996].

[6] Another set of OLR spectra, together with the broadband fluxes, is synthesized from the Geophysical Fluid Dynamics Lab (GFDL) atmospheric general circulation model (GCM) AM2 [GFDL Global Atmospheric Model Development Team, 2004], by using a $1 \mathrm{~cm}^{-1}$ nominal resolution radiation code, MODTRAN [Bernstein et al., 1996], with spatial and temporal sampling made consistent with the AIRS and CERES observations. For comparison purposes, both sets of radiance data have been convoluted into $2 \mathrm{~cm}^{-1}$ spaced frequency grids. Detailed descriptions about the configuration of the spectral simulation and the processing of the radiance data are given by Huang et al. [2007b].

\section{Results}

\subsection{Observations}

[7] Figure 1 illustrates the correlation between OLR fluxes and SST based on the time series of 16-day (orbital repeating cycle of Aqua) means at each 1 degree by 1 degree grid. The SGE regions with significant $(>95 \%$ confidence, assuming independent samples) anti-correlation between 16-day mean OLRs and SSTs are shaded. "Super" in SGE means a very strong positive (destabilizing) feedback which overrides the stabilizing Planck damping (see equation (1)). This is a different perspective from the sense of a strong greenhouse trapping which refers to surface emission minus OLR as studied by Raval and Ramanathan
[1989]. Note that most of the SGE region lies out of the area with minimum annual mean total-sky OLR fluxes (as illustrated by the $240 \mathrm{~W} \mathrm{~m}^{-2}$ contour line) and also with relatively small standard deviation (not shown). As interpreted by Bony et al. [1997] and Allan et al. [1999], such anti-correlation is largely due to these regions transitioning between ascending (convective) and descending (convection-suppressed) regimes as a consequence of the seasonal movement of the Hadley circulation. Hence, the OLR-T relationship associated with SGE is also different from that associated with climate change forced by greenhouse gases [Held and Soden, 2000].

[8] We then take the 16-day mean convoluted AIRS radiances at each $2 \mathrm{~cm}^{-1}$ interval over the oceanic SGE regions as identified in Figures $1 \mathrm{a}$ and $1 \mathrm{~b}$, and correlate them with the corresponding 16-day mean SSTs at each grid point. Figure 2 illustrates the linear-regression coefficients between the radiances and SSTs at frequencies where the correlations are significant ( $>95 \%$ confidence). Figures $3 \mathrm{a}$ and $3 \mathrm{~d}$ illustrate the variation of clear- and total-sky radiances with SST. The normalized radiance anomaly

$$
\left(\frac{\mathrm{R}_{\nu}\left(\mathrm{T}_{\mathrm{s}}^{\mathrm{i}}\right)-\frac{1}{\mathrm{n}} \sum_{\mathrm{i}}^{\mathrm{n}} \mathrm{R}_{\nu}\left(\mathrm{T}_{\mathrm{s}}^{\mathrm{i}}\right)}{\frac{1}{\mathrm{n}} \sum_{\mathrm{i}}^{\mathrm{n}} \mathrm{R}_{\nu}\left(\mathrm{T}_{\mathrm{s}}^{\mathrm{i}}\right)},\right.
$$

where $\nu$ represents different frequencies and i designates different temperature bins) is plotted as a function of SST (binned into 1-K intervals; bins with less than 10 samples are dropped) and frequency.

[9] Under clear-sky, the sign of the coefficients differs in different absorption bands, being positive over most of the window region between 900 and $1100 \mathrm{~cm}^{-1}$, and slightly 

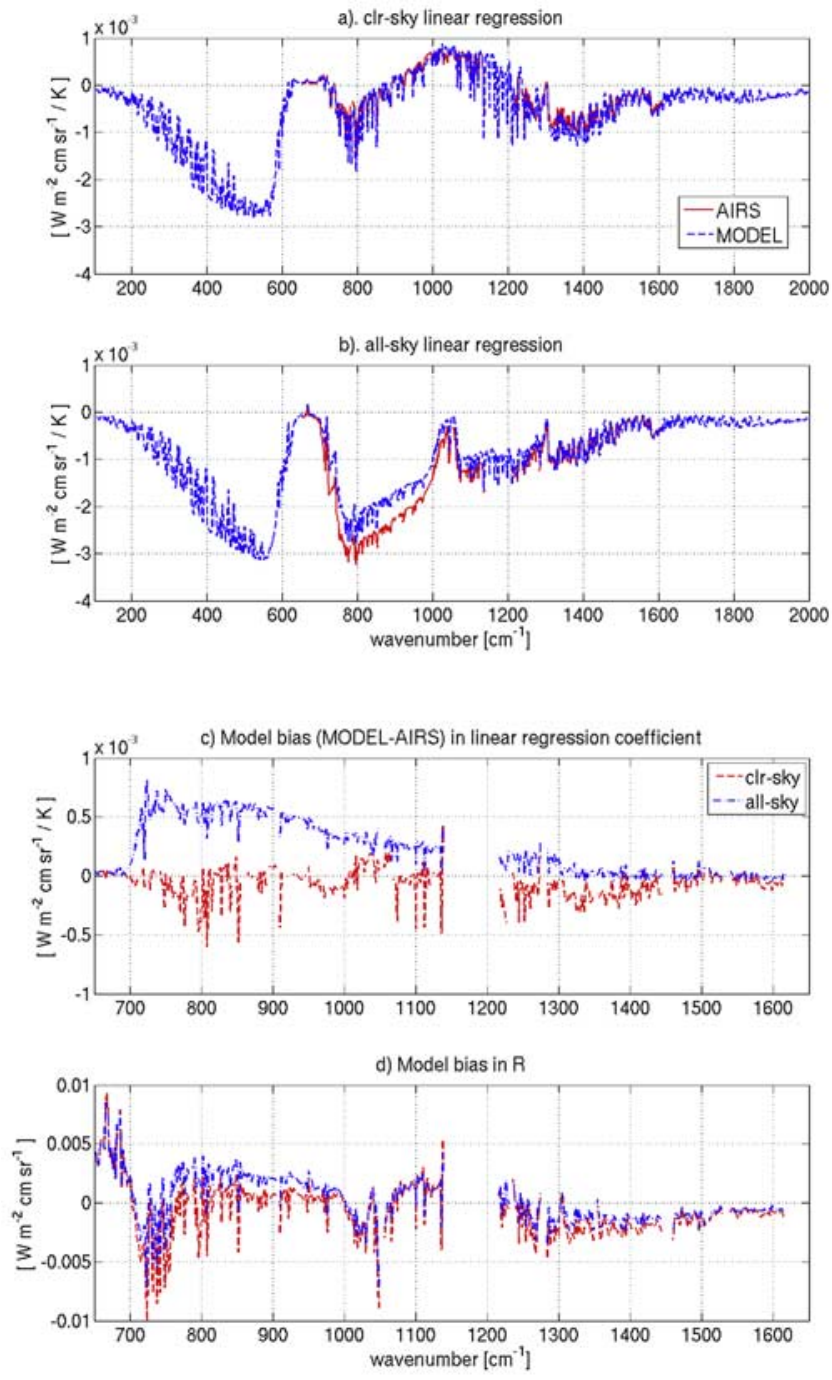

Figure 2. (a) Clear-sky and (b) total-sky linear regression coefficients of outgoing radiances to SSTs in the SGE regions (see Figure 1), (c) Model-AIRS difference in regression coefficients, and (d) Model-AIRS difference in mean radiance spectrum.

positive in the $\mathrm{CO}_{2} v 2$ band between 620 and $720 \mathrm{~cm}^{-1}$ and at $1304 \mathrm{~cm}^{-1}$ where strong carbon dioxide and methane absorption lines make it sensitive to the stratosphere and tropopause, respectively, and being negative elsewhere.

[10] The regression coefficients, with signs consistent with those of the correlation coefficients, quantify how radiation energy in each frequency interval responds to the surface temperature change. The outgoing radiance (R) at any frequency can be thought of as the sum of surface emission $\left(\mathrm{E}_{\mathrm{s}}\right)$ attenuated by the atmosphere transmission $(\mathrm{Tr})$ and the reemission by the atmosphere $\left(\mathrm{E}_{\mathrm{A}}\right): \mathrm{R}=\mathrm{E}_{\mathrm{S}} \cdot \mathrm{T}_{\mathrm{r}}+\mathrm{E}_{\mathrm{A}}$. Then, the response of $R$ to $T_{s}$ is

$$
\frac{\partial \mathrm{R}}{\partial \mathrm{T}_{\mathrm{s}}}=\frac{\partial \mathrm{E}_{\mathrm{s}}}{\partial \mathrm{T}_{\mathrm{s}}} \cdot \operatorname{Tr}+\left(\mathrm{E}_{\mathrm{s}} \frac{\partial \operatorname{Tr}}{\partial \mathrm{T}_{\mathrm{s}}}+\frac{\partial E_{A}}{\partial \mathrm{T}_{s}}\right),
$$

where the first term on the right hand side is non-negative, representing the Planck function temperature dependence; the part in brackets is usually negative because increased water vapor concentration with increase in $T_{S}$ leads to an increased atmospheric attenuation plus an equivalent lifting of atmospheric emitting level (to a colder temperature). The sum of these competing effects determines the sign of $\frac{\partial R}{\partial T_{\text {s }}}$.

[11] In the window region $\left(850-1200 \mathrm{~cm}^{-1}\right)$, although water vapor continuum absorption scales quadratically as water vapor concentration, the overall weak absorption (large Tr) makes the first term in the above equation dominate, so that the compensation of the terms does not render an anti-correlation between outgoing radiation and surface temperature. Most of the SGE arises from the water vapor vibration-rotational $(v 2)$ band (beyond $1250 \mathrm{~cm}^{-1}$ ) and the rotational band $\left(200-560 \mathrm{~cm}^{-1}\right.$, see the discussion in the next subsection) where $\operatorname{Tr}$ is very small, with substantial contributions from the wings of $\mathrm{CO}_{2} v 2$ band $\left(560-620 \mathrm{~cm}^{-1}\right.$ and $720-900 \mathrm{~cm}^{-1}$ ). Taking into account the spectral sensitivity kernels of the OLR [Huang et al., 2007a, Figure 2], it is inferred that the change of upper- and middle-tropospheric water vapor, which influences the radiances in these spectral regions, is the major contributor to the SGE. This conclusion, independently drawn from the spectral observations, reiterates the findings based on numerical perturbation experiments by Allan et al. [1999].

[12] For total-sky, except in the band center $\left(667 \mathrm{~cm}^{-1}\right)$ of the $\mathrm{CO}_{2} v 2$ band, the correlation is negative throughout the spectrum. Compared to the clear-sky condition, besides an intensification of SGE in the water vapor bands, the anticorrelation extends to the window region as well. Clouds, especially the ones at high altitudes, intercept outgoing longwave radiation at nearly all infrared frequencies and reemit at lower temperatures, thus yielding a strong greenhouse effect. Moreover, it is evident from these results that the clouds in the SGE regions respond to the SST variations coherently with water vapor, i.e. when SST warms the increase of interception exceeds the increase of surface emission. From the regression coefficients, the window region contribution due to clouds' role now overrides the water vapor $v 2$ band and becomes comparable with that of the rotational band (made evident by the simulations discussed below).

\subsection{Simulation}

[13] The correlation between the AM2 simulated OLR fluxes and SSTs closely resembles the observed pattern for both clear- and total-skies (Figure 1), thus confirming the GCM's ability to reproduce the SGE phenomenon, as shown for the reanalysis model [Allan et al., 1999]. Then, the correlation and regression analyses are applied to the simulated radiances. The simulation results are compared to the observations in Figures 2 and 3.

[14] The simulation results ranging from 100 to 2000 $\mathrm{cm}^{-1}$ extend to the far infrared regions and thus cover the entire longwave spectrum unlike the AIRS spectrum. At most frequencies where AIRS observations are available, the sign of the correlation between simulated radiances and SSTs is consistent with the observation, varying with different absorption bands for clear-sky but remaining negative nearly throughout the spectrum for total-sky. One noticeable difference is in the window region (833$1250 \mathrm{~cm}^{-1}$ ) in the clear-sky case, where simulated correlations show a more pronounced variation with frequency than AIRS and differ in sign at a few frequencies, e.g. at 976 

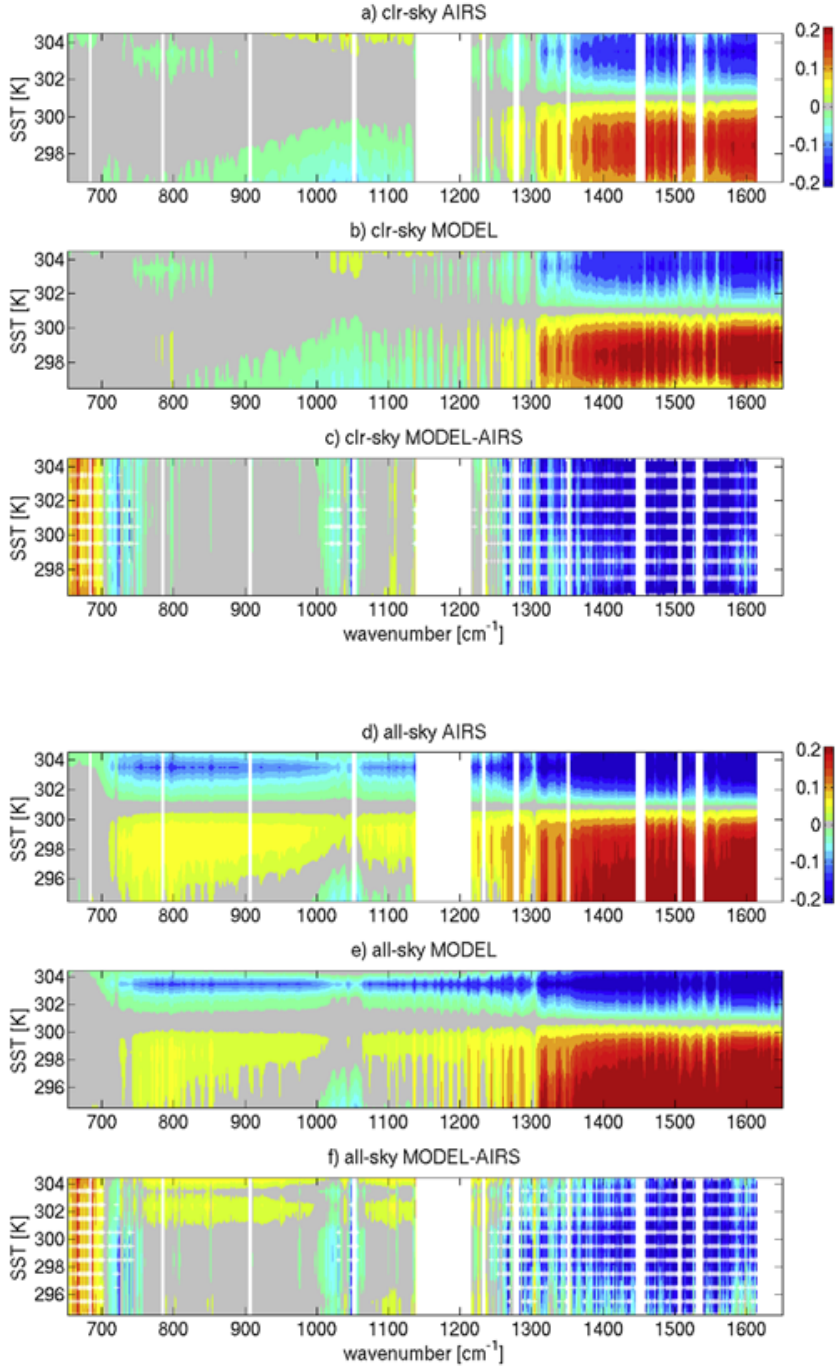

Figure 3. Longwave radiance variation with SST. Clearsky normalized radiance (dimensionless) anomalies in (a) AIRS spectra and (b) MODEL spectra. (c) Stratification of MODEL-AIRS bias with respect to SST (running symbols denote significant difference at $95 \%$ confidence interval). (d), (e), and (f) The counterparts of Figures 3a, 3b, and 3c, respectively, for total-sky.

and $1100 \mathrm{~cm}^{-1}$, where weak water vapor lines exist. The frequencies of discrepancy being only a few suggests these may be more likely due to the subtle differences of the weighting functions between the MODTRAN simulation and the real AIRS channels at these frequencies, rather than qualitatively improper water vapor concentration response simulated in AM2.

[15] There exist prominent quantitative differences in the water vapor $v 2$ band, where the simulation yields more negative values. From the comparison in Figure $2 \mathrm{c}$, the simulated quantitative response of water vapor $v 2$ band is about $20 \%$ (relative to the AIRS observation) stronger than the observations for clear-sky. In relation to AM2's substantial underestimation of outgoing radiation in the water vapor bands (see Figure 2d) due to upper tropospheric moisture (too wet) and temperature (too cold) biases [Huang et al., 2007b; John and Soden, 2007], this model tends to overestimate not only the water vapor greenhouse effect but also its change in response to SST in the context of a seasonal cycle. This bias in the water vapor band and oscillating nature of errors in the window region compensate for the overall clear-sky OLR sensitivity to SST in the seasonal cycle as measured by the broadband irradiance flux, for which the model yields $-2.1 \mathrm{~W} \mathrm{~m}^{-2} / \mathrm{K}$ compared to $-2.3 \mathrm{~W} \mathrm{~m}^{-2} / \mathrm{K}$ from CERES (precision varies less than $0.2 \mathrm{~W} \mathrm{~m}^{-2}$ in a seasonal cycle [Loeb et al., 2007]), a seemingly good agreement.

[16] The most pronounced discrepancy in the total-sky regression coefficients occurs in the spectral region from 750 to $1150 \mathrm{~cm}^{-1}$ (Figure 2c). There, the model underestimates the total-sky outgoing radiation response by over $20 \%$. This error explains the underestimated total-sky OLR sensitivity, for which the model yields $-6.1 \mathrm{~W} \mathrm{~m}^{-2} / \mathrm{K}$ but CERES gives $-7.2 \mathrm{~W} \mathrm{~m}^{-2} / \mathrm{K}$. The relatively better clearsky agreement and the remarkable total-sky bias in the window region are strongly suggestive that clouds cause the model error here. Hence, we see this particular GFDL GCM not only underestimates the longwave cloud forcing for the mean climate state [Huang et al., 2007b], but also underestimates the strength of longwave cloud response in the SGE region. Zhang et al. [2005] compare a group of GCMs to satellite retrieved cloud products and point out that the underestimation of seasonal variability of cloud amount in the tropics is a common model problem.

[17] Interestingly, the simulated radiance sensitivity to SST change in the water vapor band agrees well with the AIRS observations for total-sky (Figure 2c) despite the persistent negative bias in radiances (Figure 3f). However, the large $(20 \%)$ bias in water vapor radiative response, as revealed for clear-sky condition, indicates that this is due to compensating errors from too strong a water vapor-induced response and too weak a cloud-induced response.

[18] Because SGE is associated with the transition between convection-active and convection-suppressed regimes, the bias in mean radiance in either or both regimes may result in a bias in the OLR sensitivity to SST change. Recognizing that the two regimes are respectively characteristic of high or low SSTs [Bony et al., 1997], we stratify the bias in model simulated radiances with respect to SST. The normalized radiance bias

$$
\left(\frac{\mathrm{R}_{\nu}\left(\mathrm{T}_{\mathrm{s}}^{\mathrm{i}}\right)_{\mathrm{MODEL}}-\mathrm{R}_{\nu}\left(\mathrm{T}_{\mathrm{s}}^{\mathrm{i}}\right)_{\text {AIRS }}}{\frac{1}{\mathrm{n}} \sum_{\mathrm{i}}^{\mathrm{n}} \mathrm{R}_{\nu}\left(\mathrm{T}_{\mathrm{s}}^{\mathrm{i}}\right)_{\text {MODEL }}}\right)
$$

is plotted in Figures $3 \mathrm{c}$ and $3 \mathrm{f}$ for clear- and total-skies, respectively. Under clear-sky, the biases in the water vapor and carbon dioxide $\nu 2$ bands as identified by Huang et al. [2007b] are persistent regardless of SST, although the negative bias in the water vapor band is noticeably smaller at lower SSTs. Under total-sky, the large bias in the window region radiance sensitivity as shown in Figure 2c, which results in the $1.1 \mathrm{~W} \mathrm{~m}^{-2} / \mathrm{K}$ bias seen in the overall OLR sensitivity relative to the CERES observation $\left(-7.2 \mathrm{~W} \mathrm{~m}^{-2}\right.$ / $\mathrm{K}$ ), is apparently due to an overestimation of radiance under the condition of high SSTs (Figure 3f). This suggests that the smaller magnitude of the simulated total-sky radiance 
response to $\mathrm{SST}$ in the SGE regions results from the underestimate of the longwave cloud forcing in the convectively active regions.

\section{Discussions}

[19] To the best of our knowledge, our analysis provides, for the first time, an observationally-derived spectral perspective of the SGE. Spectral contributions to the total effect as demonstrated above make the roles of water vapor at different altitudes evident. Upper tropospheric water vapor contributes most to the SGE via absorption in the water vapor rotational band. The water vapor continuum absorption in window region, although more strongly dependent on water vapor concentration, is not sufficient enough to compensate the Planck damping and does not render SGE by itself. Furthermore, clouds make a substantial contribution to SGE mainly via their influence in the window region.

[20] The above analyses were first performed with the first year (368 days, i.e., 23 repeat cycles of Aqua orbit) of AIRS and CERES observations; these have been confirmed with the observation of the other years. The SGE regions, the correlation and regression coefficients from either observation or simulation, are insensitive to the choice of data periods. In terms of spectral distribution of the coefficient values, the results are also robust regardless of whether the analyses are applied to regional means or grid means (not shown).

[21] The particular GFDL GCM under examination is shown to overestimate the positive longwave radiative response of water vapor to SST but underestimate that of clouds in reproducing SGE. Compensating errors exist not only for the mean TOA radiation budget [Huang et al., 2007b] but, in addition, for its sensitivity to SST in a seasonal cycle. This means that the problem cannot be rectified by correcting either cloud or water vapor bias alone, but that there is a need to tackle both issues together. The lack of window region longwave radiation response in this model relates to an underestimation of cloud radiative trapping in the convection-active regime. It is not obvious whether this model deficiency can impact climate projections given that certain regions under climate change may exhibit a similar transition from one convection regime to the other; more research is needed to explore this point.

[22] SGE is demonstrated to be a valuable element for model verification. However, because of the different nature of SGE and greenhouse gas forced climate change, we caution that their associated feedbacks $\left(\sum \frac{\partial \mathrm{F}}{\partial \mathrm{X}_{\mathrm{j}}} \frac{\partial \mathrm{X}_{\mathrm{i}}}{\partial \mathrm{T}_{\mathrm{s}}}\right.$ in equation (1)) also differ. So, the OLR-T $\mathrm{T}_{\mathrm{S}}$ relationship, as well as the associated model deficiency, in the SGE experiment, cannot be readily generalized to the climate change problem.

[23] Constraining the model sensitivity is an urgent task facing the climate research community. The spectral breakdown of the OLR-T $T_{S}$ relationship provides a new perspective which distinguishes the roles of the different influential factors. Complementary to the examination of model simulated geophysical variables (temperature, water vapor, cloud properties, etc.), it reveals and, very importantly, quantifies the radiative impacts of model errors. These two key perspectives should be integrated for the validation and development of climate models.

[24] Acknowledgments. Yi Huang is supported by NASA Earth and Space Science Fellowship, grant NNX06AF75H. We thank Leo Donner, Xianglei Huang, Yuk Yung, and two anonymous reviewers whose helpful comments and suggestions have greatly improved the paper.

\section{References}

Allan, R. P., et al. (1999), The dependence of clear-sky outgoing longwave radiation on surface temperature and relative humidity, Q.J.R. Meteorol. Soc., 125, 2103-2126.

Berstein, L. S., et al. (1996), Very narrow band model calculations of atmospheric fluxes and cooling rate, J. Atmos. Sci., 53, 2887-2904.

Bony, S., K. M. Lau, and Y. C. Sud (1997), Sea surface temperature and large-scale circulation influences on tropical greenhouse effect and cloud radiative forcing, J. Clim., 10, 2055-2077.

Bony, S., et al. (2006), How well do we understand and evaluate climate change feedback processes?, J. Clim., 19, 3445-3482.

Chahine, M., et al. (2006), AIRS: Improving weather forecasting and providing new data on greenhouse gases, Bull. Am. Meteorol. Soc., 87, 896909 .

Clement, A. C., and B. Soden (2005), The sensitivity of the tropical-mean radiation budget, J. Clim., 18, 3189-3203.

GFDL Global Atmospheric Model Davelopment Team (2004), The new GFDL global atmospheric and land model (AM2-LM2): Evaluation with prescribed SST simulations, J. Clim., 17, 4641-4673.

Haskins, R. D., R. M. Goody, and L. Chen (1997), A statistical method for testing a general circulation model with spectrally resolved satellite data, J. Geophys. Res., 102, 16,563-16,582.

Held, I. M., and B. J. Soden (2000), Water vapor feedback and global warming, Аnпu. Rev. Energy Environ., 25, 441-475.

Huang, X., J. Farrara, S. S. Leroy, Y. L. Yung, and R. M. Goody (2002), Cloud variability as revealed in outgoing infrared spectra: Comparing model to observation with spectral EOF analysis, Geophys. Res. Lett., 29(8), 1270, doi:10.1029/2001GL014176.

Huang, Y., V. Ramaswamy, and B. Soden (2007a), An investigation of the sensitivity of the clear-sky outgoing longwave radiation to atmospheric temperature and water vapor, J. Geophys. Res., 112, D05104, doi:10.1029/2005JD006906.

Huang, Y., V. Ramaswamy, X. Huang, Q. Fu, and C. Bardeen (2007b), A strict test in climate modeling with spectrally resolved radiances: GCM simulation versus AIRS observations, Geophys. Res. Lett., 34, L24707, doi:10.1029/2007GL031409.

Iacono, M. J., and S. A. Clough (1996), Application of infared interferomometer spectrometer clear sky spectral radiance to investigations of climate variability, J. Geophys. Res., 101, 29,439-29,460.

John, V. O., and B. J. Soden (2007), Temperature and humidity biases in global climate models and their impacts on climate feedbacks, Geophys. Res. Lett., 34, L18605, doi:10.1029/2007GL030736.

Kalnay, E., et al. (1996), The NCEP/NCAR 40-year reanalysis project, Bull. Am. Meteorol. Soc., 77, 437-470.

Loeb, N. G., et al. (2007), Angular distribution models for top-of-atmosphere radiative flux estimation from the clouds and the Earth's radiant energy system instrument on the Terra satellite. Part II: Validation, J. Atmos. Oceanic Technol., 24, 564-584.

Ramanathan, V., and W. Collins (1991), Thermodynamic regulation of ocean warming by cirrus clouds deduced from observations of the 1987 El Nino, Nature, 351, 27-32.

Raval, A., and V. Ramanathan (1989), Observational determination of the greenhouse effect, Nature, 342, 758-761.

Tsushima, Y., and S. Manabe (2001), Influence of cloud feedback on annual variation of global mean surface temperature, J. Geophys. Res., 106, $22,635-22,646$

Wetherald, R. T., and S. Manabe (1988), Cloud feedback processes in a general circulation model, J. Atmos. Sci., 45, 1397-1415.

Zhang, M., et al. (2005), Comparing clouds and their seasonal variations in 10 atmospheric general circulation models with satellite measurements, J. Geophys. Res., 110, D15S02, doi:10.1029/2004JD005021.

Y. Huang, Atmospheric and Oceanic Sciences Program, Princeton University, Princeton, NJ 08544, USA. (yi.huang@noaa.gov)

V. Ramaswamy, Geophysical Fluid Dynamics Laboratory, NOAA, Princeton, NJ 08540, USA. 